\title{
OPTICAL IMAGING SPECTROSCOPY
}

\author{
(Invited)
}

\author{
Richard C. Canfield \\ Center for Astrophysics and Space Sciences \\ University of California, San Diego \\ La Jolla, California 92093, U.S.A.
}

\begin{abstract}
During the recent solar maximum the combination of imaging and spectroscopy in the visible part of the spectrum became a powerful tool for observational study of flares primarily because of the development of two-dimensional charge-coupled-device (CCD) arrays. In combination with appropriate new operational methods, this has led to the ability to observe, for the first time, the preflare and impulsive-phase physical processes associated with spatially resolved features of flare loops. As a result of concurrent theoretical developments, modeling progressed from an empirical to a physical level. This made it possible to interpret imaging spectra in terms of coronal pressure and heat flux, particle beam heating, chromospheric evaporation, and explosive chromospheric dynamics at the footpoints of flare loops.

There is clear potential for further advances in the near future, taking advantage of improvements in digital recording speed $(\sim 10$-fold), number of photosensitive elements per array ( $\sim 10$-fold), real-time data pre-reduction (potentially 10- to 100-fold), and using multiple CCD arrays. By the time of the next solar maximum I expect imaging spectroscopy to achieve spatial resolution $\lesssim 1$ arc s, temporal resolution $\lesssim 5 \mathrm{~s}$, and simultaneous critically-sampled spectroscopy of several lines and continua. As a result, we can anticipate continued increase in our understanding of the physical processes and configurations of solar flares in the chromosphere, temperature minimum region, and photosphere. Even greater progress toward a more global understanding of flares will obviously come about when we have simultaneous optical, X-ray, and $\gamma$-ray imaging spectroscopy.
\end{abstract}

\section{INTRODUCTION}

Spectroscopy is much easier in the visible part of the spectrum than in other energy ranges. High photon flux and detector efficiency allow high spatial and temporal resolution. In addition, the optically thick nature of the visible solar atmosphere permits studies of the variation of physical conditions along a line of sight. Array detectors are presently leading to a quantum jump in our ability to utilize these advantages. We are seeing the merging of the morphological information contained in monochromatic $\mathrm{H} \alpha$ filtergrams and the physical information contained in spectra, in the form of what is called optical imaging spectroscopy. In this paper I will review the technological and operational advances that have enabled this advance, some of the physical understanding that has come about as a result, and the possibilities for the future. I will deliberately not describe some of the alternate uses of CCD arrays that are also attractive, such as their use with monochromatic filters to achieve high spatial or temporal resolution, at the sacrifice of spectroscopy. 


\section{OBSERVATIONAL METHODS}

The observational methods of imaging spectroscopy are very simple. A two-dimensional array is placed at a focal plane of a slit spectrograph. One of the two dimensions of the array is oriented parallel to the spectrograph slit, so the pixels in this dimension sample a line of solar image elements, i.e., spatial variations. The other dimension of the array is then parallel to the spectral dispersion, and thus samples a line of spectral elements, i.e., the spectral line profile of a single spatial image element. The array is exposed, read, digitized, and recorded, producing an observation with one spatial and one spectral dimension; all samples from a single exposure of the array are simultaneous. A second spatial dimension is built up, in the manner of a traditional spectroheliogram, by scanning the solar image perpendicular to the spectrograph slit. Observations of the preflare and impulsive phases are ensured by taking data continuously in this manner, saving the data only if a flare occurs within the scan area.

Of course, reality forces various unsatisfying tradeoffs, which are well illustrated by the $\mathrm{H} \alpha$ imaging spectroscopy program Todd Gunkler and I carried out in 1980 at the Vacuum Tower Telescope at Sacramento Peak, in coordination with the Solar Maximum Mission (Acton et al., 1982; Gunkler et al., 1984). The worst bottleneck in our 1980 observing setup was real-time digital data recording on conventional 1/2 in. magnetic tape. To deal with this relatively slow data recording it was necessary to record only $50 \times 100$ elements of the array and to slow the observational cadence to four slit-position/read/write cycles per second. This, coupled with the need to cover a reasonable area to ensure preflare converage of the flare site, forced rather coarse spatial resolution, only $\sim 2.5$ arc s per pixel. A square 50 x 50 pixel spatial image, corresponding tô 50 slit positions, required $12.5 \mathrm{~s}$ to record; slewing back to the starting point for the next scan brought the full cycle time to $15 \mathrm{~s}$. Though this cycle time was much longer than desired, it at least permitted a few samples during the impulsive phase of a typical hard X-ray flare.

Our observational approach has placed strong emphasis on spectra, at the expense of imaging. All 100 spectral data points in each spatial pixel were sampled simultaneously. Also, though our spatial and temporal resolution were far less than optimal, our spectral resolution was quite sufficient; each spectral pixel was $\sim 100 \mathrm{~mA}$ wide, which compares favorably with the $283 \mathrm{~m} \AA$ thermal Doppler width of $\mathrm{H} \alpha$ at $10^{4} \mathrm{~K}$. This emphasis on spectroscopy produced a unique new perspective on a variety of flare processes in the chromosphere, including the effects of coronal pressure, electron-beam heating, chromospheric evaporation, and flare dynamics.

\section{USING CHROMOSPHERIC H $\alpha$ PROFILES TO MEASURE CORONAL PRESSURE}

Theoretical calculations have shown that the profile of the $\mathrm{H} \alpha$ line from hydrostatic model atmospheres is a useful diagnostic of coronal pressure (Canfield, Gunkler, and Ricchiazzi, 1984, henceforth CGR). The top panel of Figure 1 shows four different theoretical $\mathrm{H} \alpha$ profiles, computed from physical model atmospheres based on four different values of coronal pressure, $P_{0}$, in an idealized case in which the electron-beam heating, $\mathrm{F}_{20}$, and transition-region conductive heat flux, $\mathrm{F}_{5}$, are artificially held fixed. As pressure increases, both the total amount of $\mathrm{H} \alpha$ emission and its profile change. The total amount of emission increases with pressure both because there are more $\mathrm{H}$ atoms in the chromosphere and because the amount of $\mathrm{H} \alpha$ radiation per $\mathrm{H}$ atom increases. The line loses its central reversal because at high pressures, more frequent collisions of $\mathrm{H}$ atoms with free electrons tie the line emissivity more closely to temperature, which increases 
monotonically outward in the chromosphere. CGR showed that although the total amount of emission is not a unique signature of coronal pressure, the relative strength of the central reversal is; only high coronal pressures $\left(\gtrsim 10^{3}\right.$ dynes $\left.\mathrm{cm}^{-2}\right)$ produce $\mathrm{H} \alpha$ profiles that are not centrally reversed.

A typical observed spectroscopic manifestation of the increase to high values of coronal pressure in the course of a flare is seen in Figure 2, which shows time sequences (15 s apart) of observed $\mathrm{H} \alpha$ profiles of two interesting pixels of one of the two 1980 flares that were well observed (i.e., imaging spectroscopy in $\mathrm{H} \alpha$, soft $\mathrm{X}$ rays, and hard X rays) from both SMM and Sacramento Peak (the flare of $\sim 1522$ UT, 14 June 1980; Gunkler et al., 1984). The north pixel is typical of the impulsively-brightening north region of this flare, and the strand pixel typifies an $\mathrm{H} \alpha$ ribbon. The times of $\mathrm{H} \alpha$ observations during the period of impulsive hard X-ray emission are underlined; 28 to $54 \mathrm{keV} \mathrm{X}$ rays peaked at $\sim 1523$ UT. Comparison with the CGR theoretical $\mathrm{H} \alpha$ profiles suggests that, in the north pixel, the value of the pressure increased throughout the impulsive phase and remained at a high level, between $10^{2}$ and $10^{3}$ dynes $\mathrm{cm}^{-2}$, immediately afterward. In the strand pixel, the comparison suggests that pressure was already at a high value by the time of hard X-ray peak, and also remained at such values $\left(\sim 10^{3}\right.$ dynes $\left.\mathrm{cm}^{-2}\right)$ immediately thereafter.

Comparison of pressure values at the times of maximum thermal X-ray emission measure of both well-observed 1980 flares (1456 UT, 7 May and 1522 UT, 24 June) has demonstrated pressure equilibrium between the coronal parts of flares and their chromospheric footpoints, as one would expect from a loop flux-tube visualization of flare geometry (Gunkler et al., 1984; Canfield and Gunkler, 1985). Puzzling earlier indications of pressure imbalance between the chromosphere and corona (Acton et al., 1982) were shown by Canfield and Gunkler (1985) to be invalid, due to the use of physically incomplete empirical models rather than physical models for the theoretical interpretation of the observed $\mathrm{H} \alpha$ profiles.

\section{CHROMOSPHERIC FOOTPOINT PENETRATION OF NONTHERMAL ELECTRONS}

$\mathrm{H} \alpha$ line profile calculations based on theoretical hydrostatic model atmospheres indicate that there is a unique signature of intense nonthermal electron heating by Coulomb collisions, in addition to the pressure signature discussed above. The lower panel of Figure 1 shows three different theoretical $\mathrm{H} \alpha$ profiles for physical model atmospheres based on three different values of $F_{20}$, the energy flux of nonthermal electrons above $20 \mathrm{keV}$. As $F_{20}$ is increased, holding $F_{5}$ and $P_{0}$ constant, substantial wings of distinctly non-Gaussian form appear. This effect is due to linear Stark broadening of the upper and lower atomic levels of the H $\alpha$ transition; Stark broadening has a much different spectral form in the wings than the Gaussian form appropriate to thermal broadening. This Stark-broadened wing emission arises relatively deep in hydrostatic chromospheres, where the density is large enough that Stark broadening becomes significant. CGR showed that such obvious Stark wings, having form much different from the more Gaussian form shown in the top panel, and extending $>3 \AA$ from line center, are a unique signature of intense electron beams $\left(\mathrm{F}_{20} \gtrsim 10^{10} \mathrm{erg} \mathrm{cm}^{-2} \mathrm{~s}^{-1}\right)$.

Canfield, Gunkler, and Kiplinger (1984), studying the two well-observed 1980 flares, found direct spatial correspondence between $\mathrm{H} \alpha$ Stark-wing sites and the strongest $\gtrsim 16 \mathrm{keV}$ emission sites observed by the Hard X-Ray Imaging Spectrometer (HXIS). Hard X-ray and H $\alpha$ Stark-wing sites from both flares were also well correlated in time with higher-energy $\mathrm{X}$ rays observed by the 
Hard X-Ray Burst Spectrometer (HXRBS). Figure 3 shows hard X-ray counts for these two flares, and allows a comparison of their temporal variations with those of typical H $\alpha$ kernel (Stark-wing) pixels. Ignoring, for the moment, the $\mathrm{H} \alpha$ asymmetry (red excess), there is a clear tendency for the $\mathrm{H} \alpha$ line wings to broaden and narrow in close synchronism with the hard X-ray count rate while, for example, the $\mathrm{H} \alpha$ line center intensity varies both more slowly and qualitatively differently. The obvious conclusion is that nonthermal particles not only stop at the footpoints of loops, as also indicated by hard X-ray imaging of impulsive flares, but actually penetrate into the chromosphere.

It is interesting that when observations of the extent of the $\mathrm{H} \alpha$ wing broadening are used to estimate the value of $F_{20}$, one gets high values of $F_{20}$ in the brightest kernels of both wellobserved 1980 flares. These values, $F_{20} \sim 10^{11} \mathrm{erg} \mathrm{cm}^{-2} \mathrm{~s}^{-1}$, are very close to what one would infer to be the limiting electron beam flux permitted by reverse current stability considerations. Moreover, $F_{20}$ values of the same order are inferred for these events if $F_{20}$ is calculated by a quite independent method, using the total flare power inferred from the spatially unresolved HXRBS data and the observed H $\alpha$ Stark-wing kernel area. This suggests that our $1980 \mathrm{H} \alpha$ spatial resolution, though only $\sim 2.5$ arc $\mathrm{s}$, is probably within an order of magnitude of what is really necessary to properly resolve the true $\mathrm{H} \alpha$ kernel image.

\section{CHROMOSPHERIC EVAPORATION: BEAMS OR CONDUCTION?}

A topic of considerable current interest is chromospheric evaporation, which is the process of heating chromsopheric material to typical flare coronal temperature $\left(\mathrm{T}>10^{6} \mathrm{~K}\right)$. Among the issues of debate (cf. Doschek et al, 1985) are: (1) Can chromospheric evaporation account for the amount of X-ray emitting material in flares; (2) What is the mechanism that drives chromospheric evaporation?

Various approaches have been taken to answering the first question, including the use of $\mathrm{H} \alpha$ imaging spectroscopic observations. Acton et al. (1982) compared $\mathrm{H} \alpha$ imaging spectra of the wellobserved 7 May 1980 flare to theoretical $\mathrm{H} \alpha$ profiles based on empirical model flare chromsopheres. On the basis of the relative strength of the central reversal, Acton et al. were able to assign to each pixel in the $\mathrm{H} \alpha$ image an amount of material, per pixel, that had been evaporated. Integrating over the flare area, they compared this quantity to that implied by the soft X-ray observations, and found that the $\mathrm{H} \alpha$ profiles implied the evaporation of more than enough material to account for the observed X-ray emission measure and image size. A less empirical approach, based on the physical models of CGR, was later taken by Canfield and Gunkler (1985), in the course of resolving the controversy over pressure imbalance alluded to in Section III above. However, because this approach treats coronal pressure and transition region conductive flux as independent parameters (although they are certainly not), it is capable of observationally confirming only the presence of high pressure, but not its physical cause. Hence, Canfield and Gunkler were able to show, on simple physical grounds based on coronal loop equilibrium scaling laws, only that conductively-driven evaporation could theoretically account for the inferred amount of X-ray emitting material.

The main contribution of $\mathrm{H} \alpha$ imaging spectroscopy to the second question comes about because this method of observation gives information about both the spectrum and the image morphology. As discussed in Section IV above, this allows the observational determination of the electron beam energy flux $F_{20}$. In the two well-observed 1980 flares Gunkler et al. (1984) and Canfield and Gunkler (1985) determined $F_{20}$ in order to estimate the amount of chromospheric evaporation that arose due to direct Coulomb heating by the beam electrons, which they found to be less than the amount of chromospheric evaporation due to classical thermal conduction, by the time of peak X-ray emission measure. 


\section{CHROMSOPHERIC RADIATING SHOCKS AND CONDENSATIONS}

Our theoretical understanding of the dynamics of the atmosphere contained in flare loops has progressed dramatically during this solar maximum. The most novel physics lies in the understanding of chromospheric aspects of explosive evaporation driven by intense impulsive electron beams, which initiate downward-propagating radiating shocks (Fisher, 1985) at the leading edge of dense chromospheric condensations (Fisher, Canfield, and McClymont, 1985). These chromospheric phenomena may have the same origin as the impulsive-phase X-ray blue-shifts seen from SMM, P78-1, and Hinotori (Doschek et al., 1985), though they certainly involve much different physics. Physical understanding of chromospheric condensations is so recent that there do not yet exist theoretical $\mathrm{H} \alpha$ line profiles with which to compare the observations. However, physical conditions in strong condensations are known, as shown in Figure 4, which focuses on the upper chromospheric part of a flare loop during the first few seconds of heating by an intense impulsive electron beam. To the left of the dashed vertical line, in all panels of the figure, is the hightemperature plasma that is thought to be the source of the blue-shifted X-ray emission. Between the dashed and solid vertical lines is the condensation itself, which is somewhat cooler and much denser than its surroundings, and is moving downward at velocities (initially approaching $100 \mathrm{~km}$ $\mathrm{s}^{-1}$ ) that far exceed the sound speed in the underlying electron-beam-heated upper chromosphere. Fisher (1985) has recently developed a simple model of a radiating shock and condensation propagating into an isothermal chromosphere, which predicts that the timescale for disappearance of the downward motions should be $\sim 1 \mathrm{~min}$, independent of the strength of the condensation. Straightforward physical arguments, supported by the numerical simulations, indicate that explosive evaporation should take place only for $F_{20} \gtrsim 10^{10} \mathrm{erg} \mathrm{cm}^{-2} \mathrm{~s}^{-1}$ (Fisher, Canfield, and McClymont, 1985); since the observations discussed above imply such large values of $F_{20}$ in flare kernels, we should expect to see spectroscopic evidence of chromospheric condensations.

The five 1980 flares for which we have both imaging spectroscopy in H $\alpha$ and non-imaging spectroscopy in hard X-rays (from HXRBS on SMM) show properties that are very suggestive of chromospheric condensations driven by explosive chromospheric evaporation and electron beam heating (Canfield, Gunkler, and Kiplinger, 1985). One or more pixels of all five flares show the $\mathrm{H} \alpha$ Stark-wing signature. These pixels have several interesting properties in difference spectra, which are formed by subtracting their pre-impulsive spectrum, as shown in Figure 5. First, during the impulsive phase redshifts are ubiquitous; peak-observed Doppler velocities for all five events are typically 40 to $60 \mathrm{~km} \mathrm{~s}^{-1}$ downward. Multiple redshift episodes in the H $\alpha$ spectrum can of ten be identified with major impulsive spikes in the hard X-ray count rate, as indicated in the figure. It is not uncommon for redshift episodes to decay to essentially zero in 30 to $60 \mathrm{~s}$, though more long-lived redshifts (probably multiple episodes) are also often seen. It is often observed that in the wings of $\mathrm{H} \alpha$ the Doppler shift becomes independent of intensity, indicating either that the moving material becomes optically thin or that it is optically thick, but moving at the same velocity over a broad range of column depth. Often the excess spectrum is either weakly centrally reversed or not reversed at all, indicating high pressure.

During the time of the balloon flight that discovered hard X-ray microflares (Lin et al., 1984), we were making $\mathrm{H} \alpha$ imaging spectroscopic observations at Sacramento Peak. Canfield and Metcalf (1984) have found that the $\mathrm{H} \alpha$ counterparts of the larger observed hard X-ray microflares have bright kernels that show impulsive-phase $\mathrm{H} \alpha$ wing broadening that rises and falls in synchronism with hard X-rays, indicative of chromospheric electron-beam heating. The flare-excess emission of these larger microflares is clearly redshifted, with Doppler velocities that are typically $\sim 50 \mathrm{~km} \mathrm{~s}^{-1}$. Both the impulsive-phase wing broadening and the redshifts indicate that although these microflares are small in area, they still have intense electron beams with $F_{20} \gtrsim 10^{10}$ erg $\mathrm{cm}^{-2} \mathrm{~s}^{-1}$. Metcalf and Canfield (1985) have examined the $\mathrm{H} \alpha$ counterparts of the smallest 
microflares observed in hard $\mathrm{X}$ rays. They find that in them there is little or no $\mathrm{H} \alpha$ redshift. It is interesting that they still show evidence of electron beams, however, although with $F_{20} \lesssim 10^{10} \mathrm{erg} \mathrm{cm}^{-2} \mathrm{~s}^{-1}$. In particular, they show weak $\mathrm{H} \alpha$ wing enhancements temporally correlated with hard $\mathrm{X}$ rays, and line profiles much like the theoretical profiles for low-pressure atmospheres heated by weak electron beams shown in Figure 1 (upper panel, $P_{0}=1-10$ dynes $\mathrm{cm}^{-2}, \mathrm{~F}_{20}=10^{8} \mathrm{erg} \mathrm{cm}^{-2} \mathrm{~s}^{-1}$ ). Finally, there is evidence for $\mathrm{H} \alpha$ events much like the $\mathrm{H} \alpha$ counterpart of the smallest hard X-ray microflares, but without detected hard X-ray counterparts, supporting the suggestion of Lin et al. (1984) that the distribution of non-thermal events extends to very small events indeed.

\section{FUTURE PROSPECTS FOR THEORY AND OBSERVATION}

One of the delights of working on impulsive-phase chromospheric flare physics at this time is that both theory and observation are advancing at a rapid pace.

On the theoretical side, it is clear that more work needs to be done to complete our physical understanding of the relationship between the properties of the mechanism that leads to explosive evaporation and the properties of the dynamic phenomena that ensue. For example, to what extent will a coronal conduction front lead to explosive evaporation, creating a chromospheric condensation? How is the velocity (or temperature density) of a condensation related to $\mathrm{F}_{20}$ ? What effects will return currents or beam instabilities have? Second, we need to predict specific observable spectral parameters of dynamic atmospheres, much as has already been done for $\mathrm{H} \alpha$ from static atmospheres. How does the $\mathrm{H} \alpha$ profile reflect the presence of radiating shocks and condensations? How about transition-region lines, or lines formed deeper in the chromosphere? How much emission comes from the radiating shock and condensation, as compared with the underlying atmosphere that has not yet been shocked? How can simultaneous observations of more than one line, formed at different temperatures, discriminate between various transport processes?

On the observational side, it is obvious that during the next solar maximum we will be able to do dramatically better than what we did during the past one. We can estimate the quantitative improvements by assuming that we will use devices that are available right now. A major advance can certainly come from using analog-to-digital conversion and video recording hardware that has been developed for the home hi-fi market, rather than conventional $1 / 2$ in. computer tape as we did at Sacramento Peak in 1980. Presently available video-digital interfaces for home hi-fi run at 1.6 megabits per second for each (stereo) channel, and retail for about $\$ 1000$. Add a video recorder, and you can record on-line an order of magnitude faster than what we had in 1980. The number of photosites per array has also gone up about an order of magnitude over what we used in 1980. Data preprocessing, i.e., pre-reduction to reduce the number of bits actually written to tape, is now in daily use with the Multiple Diode Array (MDA) at Sacramento Peak. In principle, this will allow speed enhancements that might easily be 1 to 2 orders of magnitude. Conservatively, I estimate the potential benefit at a factor of 3 , based on setups we have developed wiht the MDA in the last year, which simply reduce the effective spectral resolution in the wings of the $\mathrm{H} \alpha$ line to compensate for the effectively slower spectral variation of the absorption coefficient there. Finally, multiple arrays, each with its own recorder, can potentially enable one to make imaging spectroscopic observations of perhaps 10 different spectral features simultaneously, using an echelle spectrograph like the one at Sacramento Peak.

Combining all these improvements, by the time of the next maximum, I expect to be able to carry out imaging spectroscopic observations, critically resolved spectroscopically, that achieve both spatial resolution $\lesssim 1$ arc $\mathrm{s}$ and temporal resolution $\lesssim 5 \mathrm{~s}$, simultaneously, for many 
spectral lines and continua formed at different temperatures or depths within the chromosphere, temperature minimum, and photosphere. However, only through imaging spectroscopy of higherenergy radiation such as microwaves, $\mathrm{X}$ rays, and $\gamma$-rays can we hope to build a compelling physical picture of the whole flare process, throughout the solar atmosphere. I look forward to the advances and challenges these new capabilities will provide for our physical understanding of acceleration and transport processes in solar flares.

Acknowledgments. During the early years of this effort our observational and theoretical research was supported by the United States Air Force Office of Scientific Research under grants 76-3071 and 82-0092, and by the National Aeronautics and Space Administration under grant NSG-7406. We are grateful for current support from the National Science Foundation; grant AST-83-20306 supports our observations, and ATM-84-15793 includes related theory. Computing facilities have been provided by the National Center for Atmospheric Research, which is supported by the National Science Foundation.

\section{REFERENCES}

Acton, L. W., Canfield, R. C., Gunkler, T. A., Hudson, H. S., Kiplinger, A. L., and Leibacher, J. W., 1982, Astrophys. J., 263, 409.

Canfield, R. C. and Gunkler, T. A., 1985, Astrophys. J., 288, 353.

Canfield, R. C. and Metcalf, T. R., 1984, Bull. Amer. Astron. Soc., 16, 891.

Canfield, R. C., Gunkler, T. A., and Kiplinger, A. L., 1984, Adv. Space Res., 4, No. 7, 255.

Canfield, R. C., Gunkler, T. A., and Kiplinger, A. L., 1985, Bull. Amer. Astron. Soc., 17, 628.

Canfield, R. C., Gunkler, T. A., and Ricchiazzi, P. J., 1984, Astrophys. J., 282, 296.

Doschek, G. A., et al., 1985, in Solar Flares: Proceedings of the SMM Workshop, eds. M. Kundu and $\mathrm{B}$. Woodgate, in press.

Gunkler, T. A., Canfield, R. C., Acton, L. W., and Kiplinger, A. L., 1984, Astrophys. J., 285, 835.

Fisher, G. H., 1985, Bull. Amer. Astron. Soc., 17, 634.

Fisher, G. H., Canfield, R. C., and McClymont, A. N., 1985, Astrophys. J., 289, 434.

Lin, R. P., Schwartz, R. A., Kane, S. R., Pelling, R. M., and Hurley, K. C., 1984, Astrophys. $J ., 283,421$.

Metcalf, T. R. and Canfield, R. C., 1985, Bull. Amer. Astron. Soc., 17, 644. 


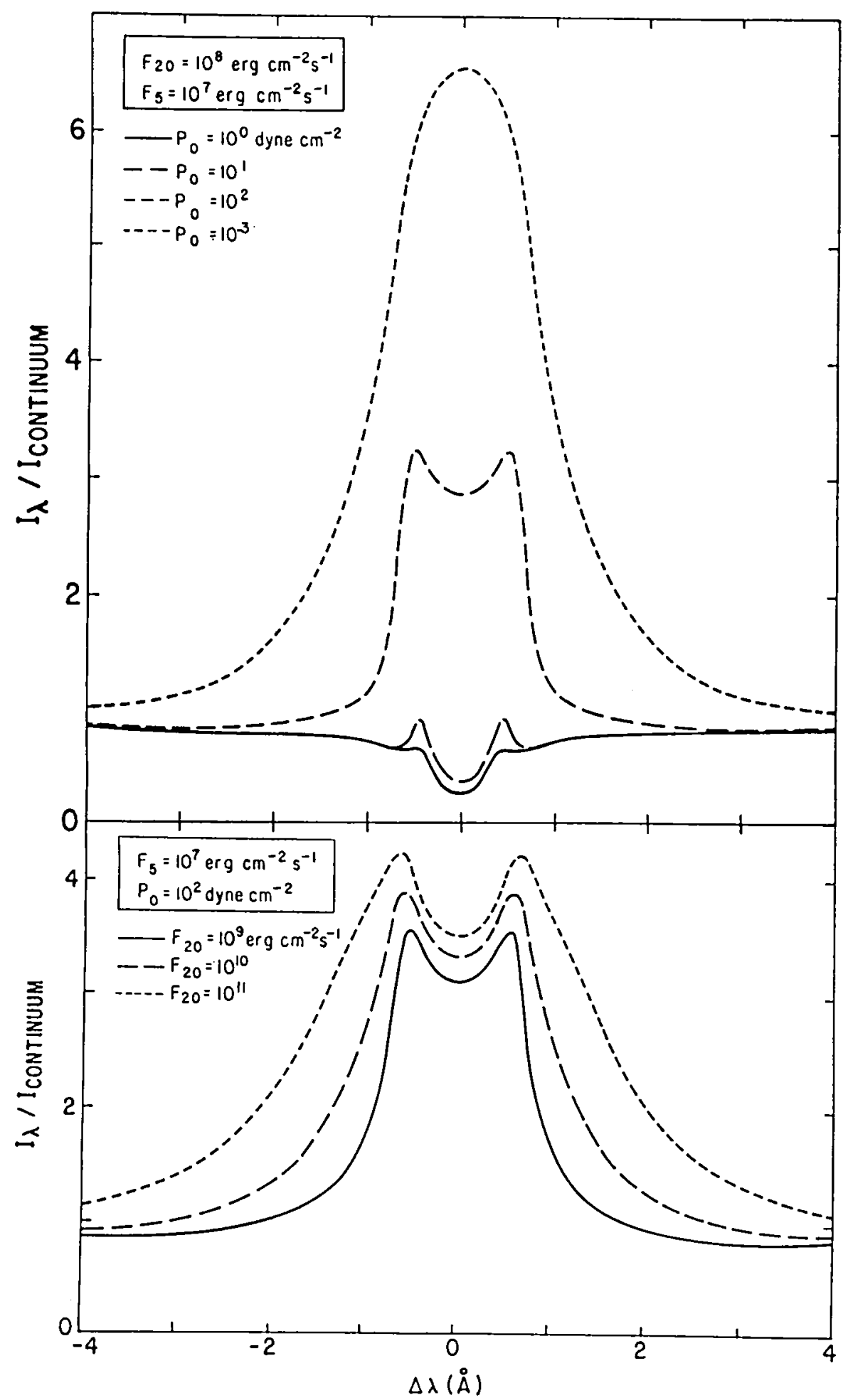

Figure 1. The effect of various physical processes on theoretical $\mathrm{H} \alpha$ profiles from hydrostatic model atmospheres. Upper panel: the effect of varying only coronal pressure $P_{0}$, holding the energy flux of nonthermal electrons $F_{20}$ and the transition-region conductive flux $F_{5}$ fixed, showing the disappearance of the central reversal in the highest-pressure model.

Lower panel: the effect of varying only $F_{20}$, holding $P_{0}$ and $F_{5}$ fixed, showing the appearance of Stark wings at high $F_{20}$. From Canfield, Gunkler, and Ricchiazzi (1984). 


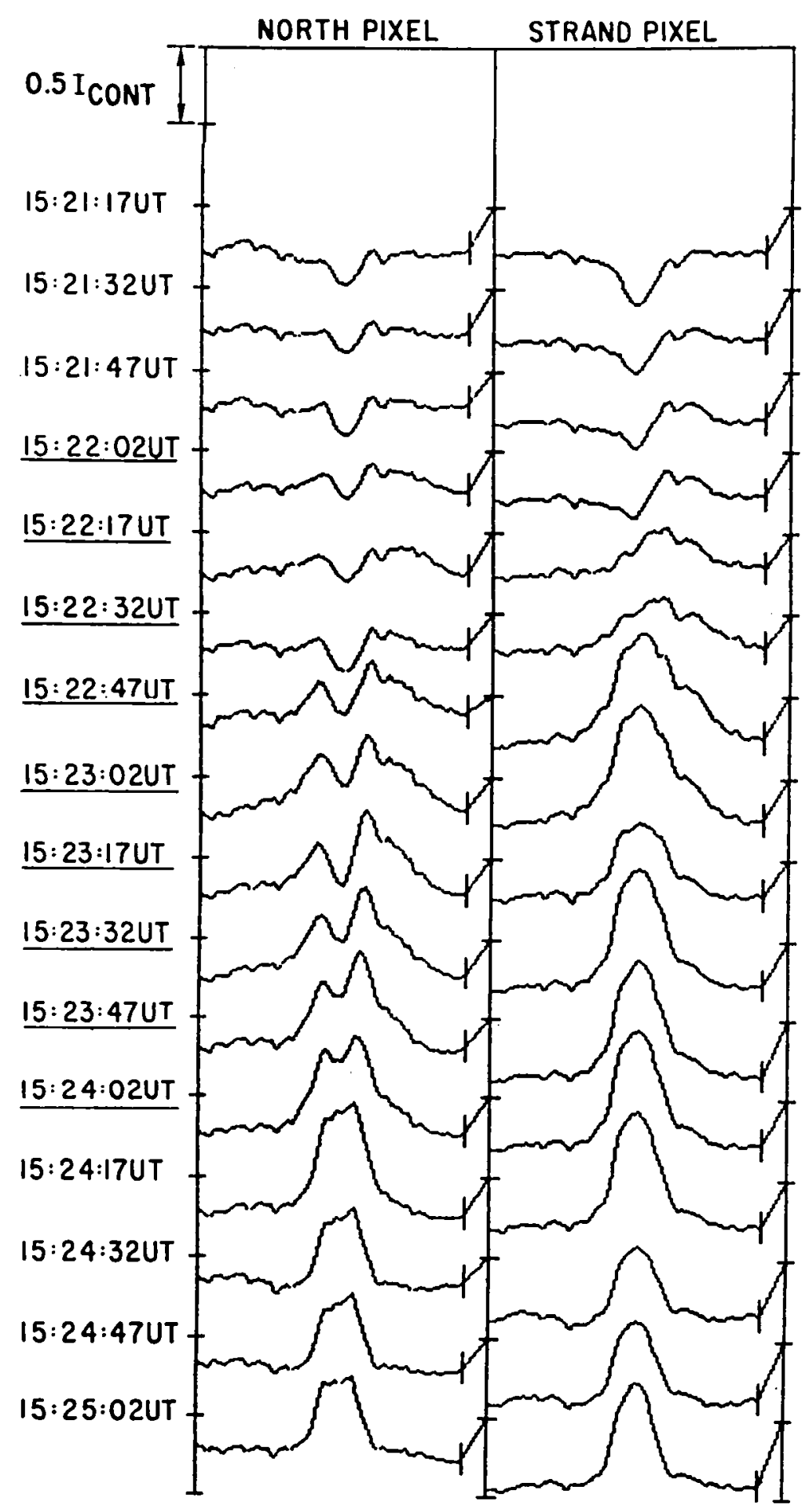

Figure 2. Observed $\mathrm{H} \alpha$ line profiles from two pixels of the flare of $1522 \mathrm{UT}, 24$ June 1980. Each column is a time sequence of profiles of a single pixel; the times given are those at which the spectrograph slit crossed the pixels shown. The spectral range of the profiles is $\mathrm{H} \alpha \pm 4.8 \AA$, and the distance between tick marks on the vertical scale is half the quiet Sun continuum intensity. To correctly identify the time associated with each $\mathrm{H} \alpha$ spectrum, note the line segment to its right linking it to its quiet Sun continuum point. From Gunkler et al. (1984). 

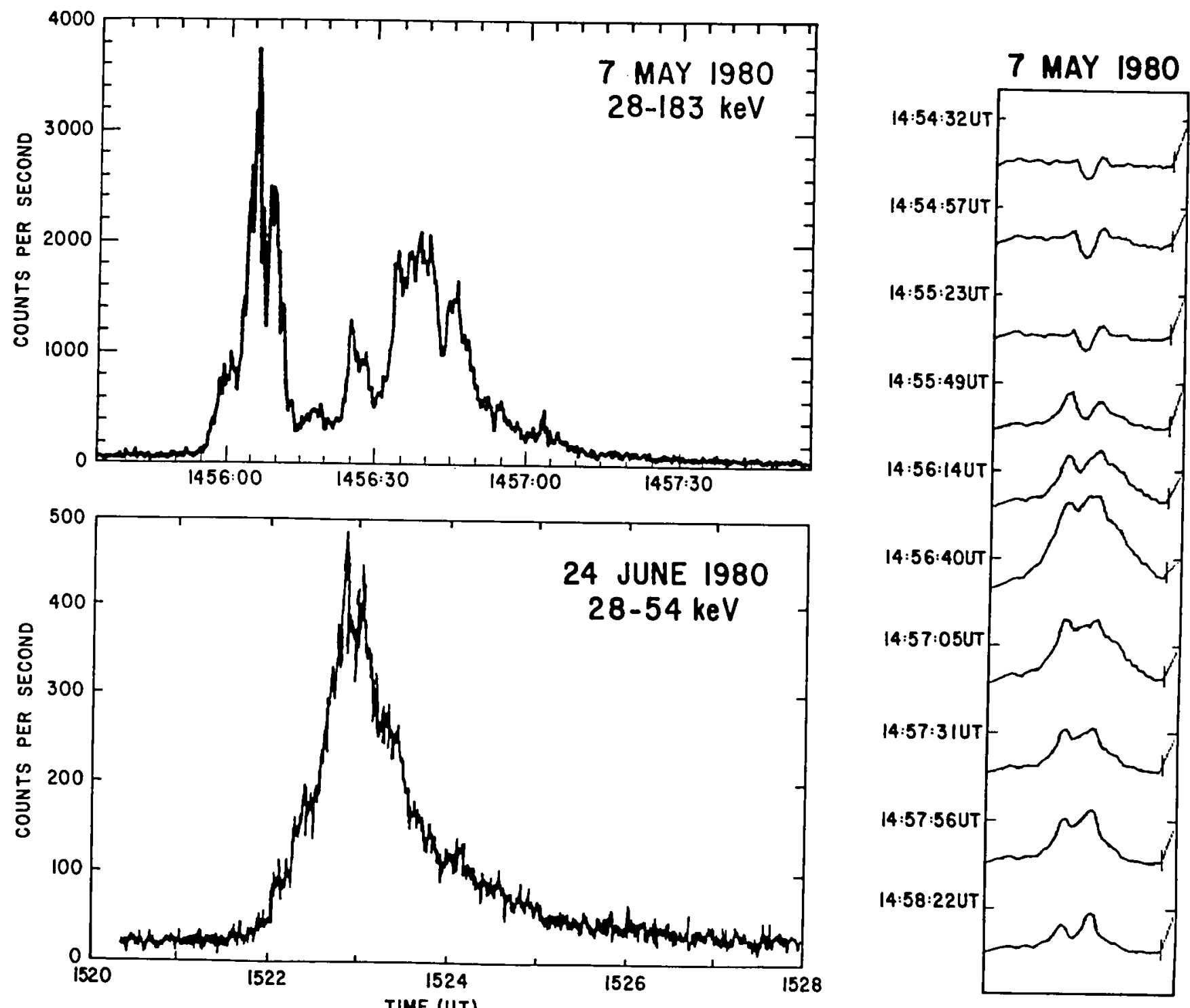

24 JUNE 1980

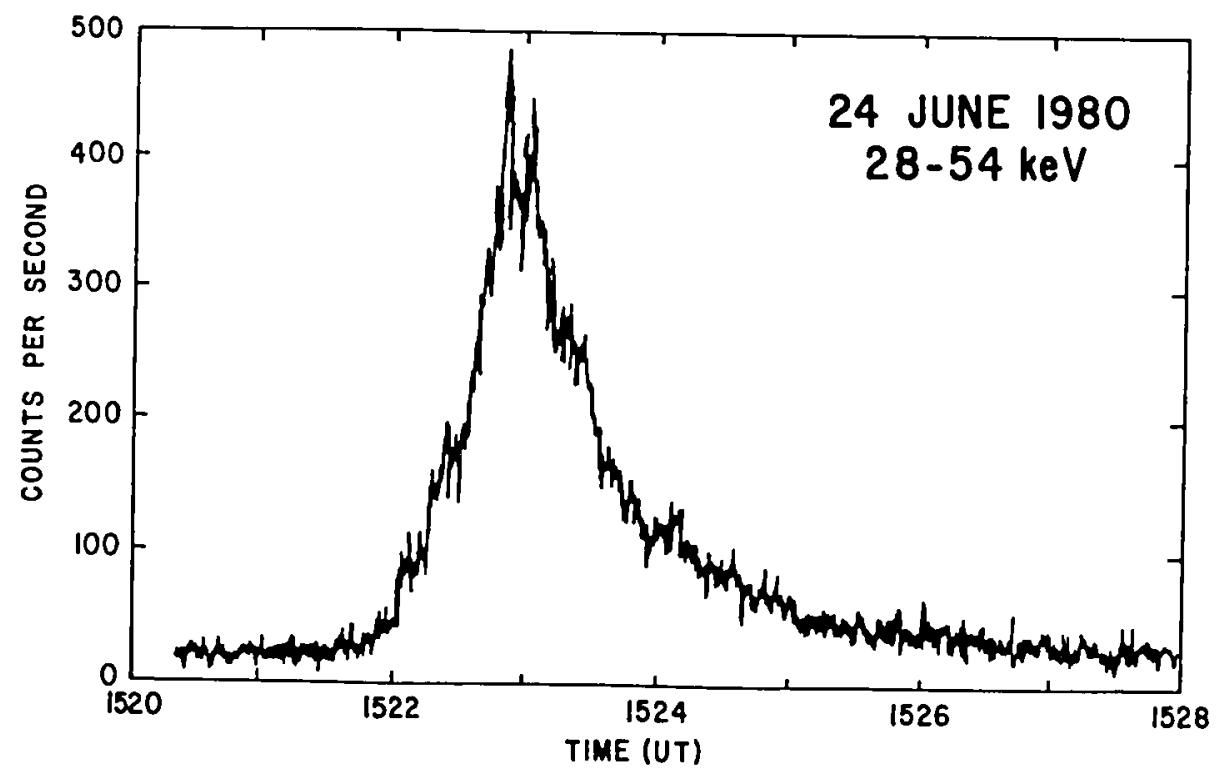

Figure 3. Hard X-ray count rates of the two 1980 flares observed well by H $\alpha$ and X-ray imaging spectroscopy and times series of $\mathrm{H} \alpha$ profiles of a typical kernel pixel in each flare. The $\mathrm{H} \alpha$ spectral scales are the same as in Figure 2. From Canfield, Gunkler, and Kiplinger (1984). 


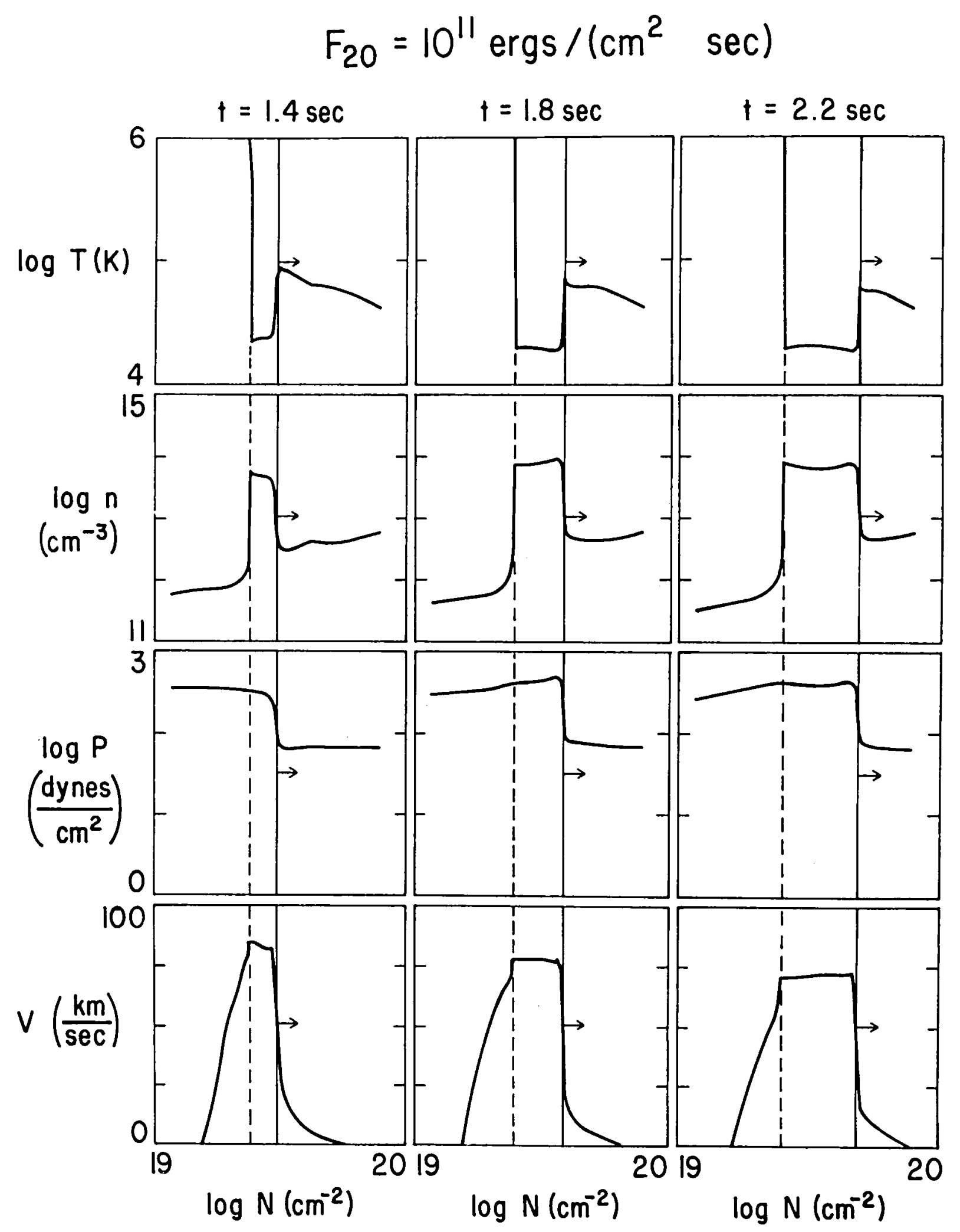

Figure 4. Computed evolution of a chromospheric condensation for the $F_{20}=10^{11} \mathrm{erg} \mathrm{cm}^{-2} \mathrm{~s}^{-1}$ flare simulation of Fisher, Canfield, and McClymont (1985). The spatial coordinate $\mathrm{N}$ is the number of hydrogen nuclei in a $\mathrm{cm}^{2}$ column, measured from the coronal loop apex. Velocity is positive in the downward direction, away from the loop apex. 

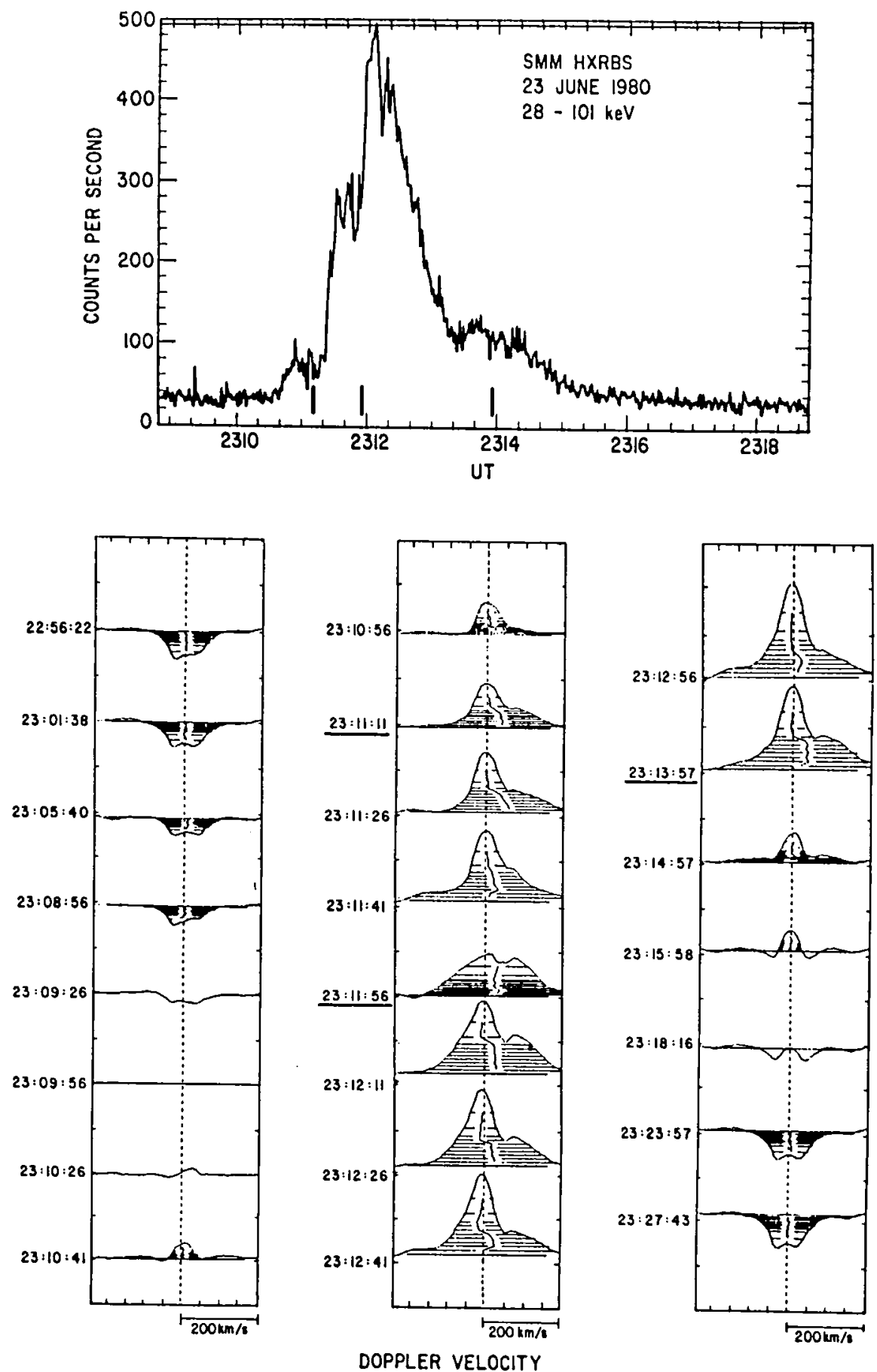

Figure 5. The relationship between (top) the hard X-ray count rate measured by the SMM Hard X-Ray Burst Spectrometer and (bottom) the $\mathrm{H} \alpha$ spectral excess (relative to its preimpulsive $\mathrm{H} \alpha$ spectrum) and Doppler shift of one of the $\mathrm{H} \alpha$ kernel pixels of the flare of 2312, UT 23 June 1980. In difference spectra of sufficient amplitude a bisector is drawn at various intensity levels indicated by horizontal striations, centered within the most intense spectral feature. Both the hard X-ray count rate and the H $\alpha$ Doppler shift show several episodes, whose occurrence is indicated by vertical bars (top panel) and underlined times of observation (bottom panel). From Canfield, Gunkler, and Kiplinger (1985). 